\title{
ON THE BRAID INDEX OF ALTERNATING LINKS
}

\author{
KUNIO MURASUGI
}

ABstract. We show that, at least for an alternating fibered link or 2-bridge link $L$, there is an exact formula which expresses the braid index $\mathbf{b}(L)$ of $L$ as a function of the 2-variable generalization $P_{L}(l, m)$ of the Jones polynomial.

\section{INTRODUCTION}

Every oriented link $L$ in $S^{3}$ is represented as a closed braid with a finite number of strings [A]. The braid index, denoted by $\mathbf{b}(L)$, of an oriented link $L$ is then defined as the minimum number of strings needed for $L$ to be represented as a closed braid. Obviously, the braid index is a link type invariant of $L$, but generally it is not easy to determine the braid index of a link.

Recently, however, some results on $\mathbf{b}(L)$ have been proved using a new polynomial $V_{L}(t)$ defined by V. F. R. Jones [J]. In fact, Jones finds a few criteria for $L$ to have $\mathbf{b}(L) \leq n$ for some $n[\mathrm{~J}]$. Shortly after the discovery of Jones' new polynomial was announced, a number of researchers (Freyd, Yetter, Hoste, Lickorish, Millet and Ocneanu [FYHLMO] and Przytycki and Traczyk [PT]) independently introduced a 2-variable polynomial $P_{L}(l, m)$ as one of the many generalizations of Jones' polynomial. This polynomial $P_{L}(l, m)$ has a more direct connection to the braid index of $L$. To be more precise, let $f(l, m)$ be a 2-variable integer Laurent polynomial, i.e., $f(l, m) \in \mathbf{Z}\left[l, l^{-1}, m, m^{-1}\right]$. Write $f(l, m)=\sum_{i=a}^{b} \phi_{i}(m) l^{i}$, where $\phi_{a}(m) \neq 0 \neq \phi_{\mathbf{b}}(m)$. We call $b-a$ the $l$-span of $f(l, m)$. Then it is proved [Mo] that

$$
l-\operatorname{span} P_{L}(l, m) \leq 2\{\mathbf{b}(L)-1\} .
$$

Jones observed in $[\mathrm{J}]$ that equality (1.1) holds for all but five knots up to 11 crossings. For alternating links it seems most likely that this is always the case, and, therefore, we propose the following conjecture.

Conjecture A. If $L$ is an alternating (nonsplit) oriented link, then

$$
l-\operatorname{span} P_{L}(l, m)=2\{\mathbf{b}(L)-1\} .
$$

Received by the editors May 11, 1988 and, in revised form, June 10, 1989. 1980 Mathematics Subject Classification (1985 Revision). Primary 57M25.

Key words and phrases. Alternating knot, alternating link, braid, Jones polynomial, braid index. This research was partially supported by NSERC Grant No. A4034. 
The main purpose of this paper is to show Conjecture A for two special but important classes of alternating links, i.e., alternating fibered links and 2-bridge links. See Theorem A and Theorem B below. Our proofs of these theorems also suggest the following slightly stronger conjecture:

Conjecture B. Let $L$ be an oriented alternating link and $L=L_{1} * L_{2} * \cdots * L_{h}$ be a *-product representation of $L$, where $L_{i}$ are special alternating links. (For a definition of the ${ }^{*}$-product, see $\S 5$ or $[\mathrm{Mu} 2]$.) Then

(1) $l$-span $P_{L}(l, m)=\sum_{i=1}^{h} l$-span $P_{L_{i}}(l, m)$.

(2) For any $i, 1 \leq i \leq h, l$-span $P_{L_{i}}(l, m)=2\left\{\mathbf{b}\left(L_{i}\right)-1\right\}$.

(3) $\mathbf{b}(L)-1$ is additive w.r.t. a ${ }^{*}$-product, i.e.,

$$
\mathbf{b}(L)-1=\sum_{i=1}^{n}\left\{\mathbf{b}\left(L_{i}\right)-1\right\} .
$$

Obviously, Conjecture B implies Conjecture A. Furthermore, Conjecture A implies another Conjecture.

Conjecture C. Let $L$ be an alternating oriented link. Then the exponent sum in a minimal braid representation is a link invariant. It will be denoted by $\mathbf{e}(L)$.

The implication Conjecture $\mathrm{A} \Rightarrow$ Conjecture $\mathrm{C}$ follows easily from (1.2) and Corollary 2 in [Mo].

In this paper, we will prove, in fact, Conjecture B and determine $\mathbf{e}(L)$ in Conjecture $\mathrm{C}$ for the alternating links mentioned earlier. (See Theorem 4.2 and Proposition 6.2.) It should be noted, however, that Conjecture B no longer holds for nonalternating links, or even for positive links. For example, the (positive) trefoil knot $K$ is a *-product of two positive Hopf links $L_{i}$. Since $l$-span $P_{L_{i}}(l, m)=2$, we have $\sum_{i=1}^{2} l$-span $P_{L_{i}}(l, m)=4$, while $l$-span $P_{K}(l, m)=$ 2 .

Conjectures A and B may be compared with the result [Mu4, Th] that, for an alternating link $L$, span $V_{L}(t)$ gives the minimum number of crossings among all the diagrams that $L$ can have. This result is no longer true if $L$ is prime and is not alternating.

Now we state the main theorems of this paper.

Theorem A. Let $L$ be an oriented alternating fibered link, and let $L=L_{1} * L_{2} *$ $\cdots * L_{h}$ be $a^{*}$-product representation of $L$, where each $L_{i}$ is an elementary torus link. Then

$$
\begin{aligned}
& \text { (1) } 2\{\mathbf{b}(L)-1\}=l \text { - } \operatorname{span} P_{L}(l, m) \text {, and } \\
& \text { (2) } \mathbf{b}(L)=h+1 .
\end{aligned}
$$

Note that $\mathbf{b}\left(L_{i}\right)=2$. 
Corollary 1. If $L$ is an oriented alternating fibered link, then the number of ${ }^{*}$-components in a reduced alternating diagram of $L$ is an alternating-link invariant. Furthermore, the numbers of positive and negative *-components of $L$ are also alternating-link invariants.

A quantity $\lambda(\widetilde{L})$ associated with a link diagram $\widetilde{L}$ is called an alternatinglink invariant if $\lambda\left(\widetilde{L}_{1}\right)=\lambda\left(\widetilde{L}_{2}\right)$ for reduced alternating diagrams $\widetilde{L}_{1}$ and $\widetilde{L}_{2}$ of the same alternating link.

If $L$ is an alternating fibered link, then $\mathbf{b}(L)$ can be described in terms of other link type invariants.

Let $c(\widetilde{L})$ denote the number of crossings in a reduced alternating diagram $\widetilde{L}$ of $L$, and let $\Delta_{L}(t)$ be the reduced Alexander polynomial of $L$. Note that $c(\tilde{L})=\operatorname{span} V_{L}(t)[\mathrm{Mu} 4]$. Then we have

Corollary 2. Let $L$ be an oriented alternating fibered link. Then, for any reduced alternating diagram $\widetilde{L}$ of $L$,

$$
\mathbf{b}(L)=c(\widetilde{L})-\operatorname{deg} \Delta_{L}(t)+1 .
$$

Proofs of Corollaries 1 and 2 will be given in $\S 7$.

The other main theorem is

Theorem B. Let $L$ be a 2-bridge link of type $(\alpha, \beta)$, where $0<\beta<\alpha$ and $\beta$ is odd. Let $\left[2 n_{1,1}, 2 n_{1,2}, \ldots, 2 n_{1, k_{1}}-2 n_{2,1}, \ldots,-2 n_{2, k_{2}}, \ldots,(-1)^{t-1} 2 n_{t, 1}\right.$, $\left.\ldots,(-1)^{t-1} 2 n_{t, k_{t}}\right]$ be a continued fraction form of $\alpha /(\alpha-\beta)$, where $n_{i, j}>0$ for all $i, j[\mathrm{~B}-\mathrm{Z}]$. Then

$$
\begin{aligned}
& \text { (1) } 2\{\mathbf{b}(L)-1\}=l \text {-span } P_{L}(l, m) \text { and } \\
& \text { (2) } \mathbf{b}(L)=\sum_{i=1}^{t} \sum_{j=1}^{k_{i}}\left(n_{i j}-1\right)+t+1
\end{aligned}
$$

Since proving Theorem $B$ is much easier than proving Theorem $A$, we will first give a proof of Theorem B in $\S \S 3-4$. A proof of Theorem A will be given in $\S \S 5-6$. Generalizing Theorem B to alternating Montesinos' links is straightforward, thus we state the formula without a proof in $\S 4$. $\S 7$ contains several consequences of these theorems. In particular, we will show that a diagram of a torus link of type $(q, r), 2 \leq q \leq r$, which is obtained from a closed $q$-braid representation, is a minimal diagram. See Proposition 7.5. In the last section $\S 8$, we will prove one theorem on $\mathbf{b}(L)$ for a special type of alternating links which was originally stated by K. Kobayashi [K2]. In the Appendix, we will prove a lemma needed in the proof of Theorem $\mathrm{A}$.

\section{Preliminaries}

In this paper, all knots or links are oriented unless stated otherwise. We begin with some notation that will be used throughout this paper.

Let $f(l, m)$ be an integer Laurent polynomial on two variables $l$ and $m$, i.e., $f(l, m) \in \mathbf{Z}\left[l, l^{-1}, m, m^{-1}\right]$. We write $f(l, m)=\sum_{i=a}^{b} \phi_{i}(m) l^{i}$, where 
$\phi_{a}(m) \neq 0 \neq \phi_{b}(m), a \leq b$ and $\phi_{i}(m) \in \mathbf{Z}\left[m, m^{-1}\right]$. Denote by $d_{\max } \phi_{i}(m)$ the maximal degree of $\phi_{i}(m)$. We define

$$
\begin{aligned}
a_{m}(f) & =d_{\max } \phi_{a}(m), \quad b_{m}(f)=d_{\max } \phi_{b}(m), \\
a_{l}(f) & =a, \quad b_{l}(f)=b, \quad \text { and } \quad l-\operatorname{span} f(l, m)=b-a .
\end{aligned}
$$

Furthermore, let $a_{f}$ and $b_{f}$ denote the coefficients of $m^{a_{m}(f)}$ and $m^{b_{m}(f)}$ in $\phi_{a}(m)$ and $\phi_{b}(m)$, respectively. We denote $A_{f}=a_{f} m^{a_{m}(f)} l^{a_{l}(f)}$ and $B_{f}=$ $b_{f} m^{b_{m}(f)} l^{b_{l}(f)}$. For convenience, we will call $A_{f}$ the minimum term and $B_{f}$ the maximum term, or the extremal terms, of $f(l, m)$.

Now, $P_{L}(l, m)$ is defined recursively as follows [L-M].

Let $L_{+}, L_{-}$and $L_{0}$ be diagrams of links which are identical except in the neighborhood of a crossing, where they look like

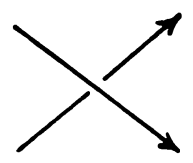

$L_{+}$

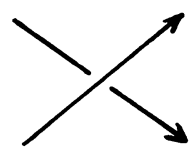

L

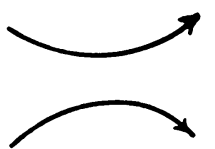

$\mathrm{L}_{0}$

Figure 1

Then $P_{L}(l, m)$ satisfies the following formula:

(1) $(1 / l) P_{L_{+}}(l, m)+l P_{L_{-}}(l, m)+m P_{L_{0}}(l, m)=0$.

(2) If $L$ is a trivial $\mu$-component link, then

$$
P_{L}(l, m)=\left(-\frac{l+l^{-1}}{m}\right)^{\mu-1} .
$$

If $f(l, m)=P_{L}(l, m)$, then $a_{f}, a_{m}(f), a_{l}(f), A_{f}, \ldots$ will be denoted by $a_{L}, a_{m}(L), a_{l}(L), A_{L}, \ldots$

Our proofs of the main theorems are based upon the precise evaluations of $A_{L}$ and $B_{L}$.

Let $L$ be a link and $\widetilde{L}$ a diagram of $L$. By changing every positive crossing $\chi^{\star}$ or negative crossing $\aleph_{\star}$ of $\widetilde{L}$ to $\asymp, \widetilde{L}$ becomes a collection of simple closed curves on $S^{2}$, each of which is called a Seifert circle of $\widetilde{L}$. (This operation $X \rightarrow \asymp$ is called a splitting.) The number of Seifert circles in $\tilde{L}$ will be denoted by $s(\widetilde{L})$.

The following proposition plays an important role in determining the braid index of a link.

Proposition 2.1 [Y]. Let $L$ be a link and $\widetilde{L}$ its diagram. Then $\mathbf{b}(L) \leq s(\widetilde{L})$.

See $[Y]$ for a proof. 
Proposition 2.2. Let $\mu(L)$ be the number of components of $L$. Then, for any diagram $\widetilde{L}$ of $L, \mu(L) \leq \mathbf{b}(L) \leq s(\widetilde{L})$.

Proof. A link $L$ of $\mu$ components cannot be represented as a closed braid with fewer than $\mu$ strings, and hence $\mu(L) \leq \mathbf{b}(L) \leq s(\widetilde{L})$. Q.E.D.

From (1.1), we see that, for any diagram $\widetilde{L}$ of $L$,

$$
l \text {-span } P_{L}(l, m) \leq 2\{s(\widetilde{L})-1\} .
$$

Proposition 2.3. Suppose that $L$ has a diagram $\widetilde{L}$ such that $l$ - $\operatorname{span} P_{L}(l, m)=$ $2\{s(\widetilde{L})-1\}$. Then $\mathbf{b}(L)=s(\widetilde{L})$.

Proof. By Proposition 2.1, $\mathbf{b}(L) \leq s(\widetilde{L})$. Suppose $\mathbf{b}(L)<s(\widetilde{L})$. Say $\mathbf{b}(L)=n$. Then $L$ has a diagram $\widehat{L}$ obtained from a natural presentation of $L$ as a closed $n$-braid. Obviously, $s(\widehat{L})=n$. Now $(2.3)$ yields $l$-span $P_{L}(l, m) \leq 2(n-1)<$ $2\{s(\widetilde{L})-1\}=l$-span $P_{L}(l, m)$, a contradiction. Q.E.D.

\section{Proof of Theorem B(I)}

As was shown in Proposition 2.3, in order to determine $\mathbf{b}(L)$, it suffices to find a diagram $\widetilde{L}$ of a 2-bridge link $L$ for which $l$-span $P_{L}(l, m)=2\{s(\widetilde{L})-1\}$. Although $L$ is an alternating link, the diagram we find need not be alternating.

Now the diagram we seek can be described most conveniently using the planar graph associated with a diagram. Therefore, we first recall the graph of a link diagram.

With a link diagram $\widetilde{L}$ of $L$, one can associate a planar graph $\Gamma$ as follows. ( $\Gamma$ will be called the graph of $\widetilde{L}$ and is denoted by $\Gamma(\widetilde{L})$.) $\widetilde{L}$ divides $S^{2}$ into finitely many domains which will be classified as shaded or unshaded. No two shaded or unshaded domains have an edge in common. Each vertex corresponds to an unshaded domain and each edge of $\Gamma$ corresponds to a crossing in $\widetilde{L}$. $\Gamma$ is weighted in the sense that each edge $e$ in $\Gamma$ is assigned the weight $w(e)=+1$ or -1 as is shown in Figure 2. $\Gamma$ depends on the shading $\rho$ of the domain in $S^{2}-\widetilde{L}$. If we use the different shading $\rho^{\prime}$, then the resulting graph is its dual with the opposite weight. (For the details, see [Mu6].) Conversely, given a weighted planar graph $\Gamma$ on $S^{2}$, one can construct a link diagram $\widetilde{L}$ of a link $L$ such that $\Gamma$ is the graph of $\widetilde{L}$. There is, however, no way to specify the orientation of $L$, and, hence, the link constructed from $\Gamma$ is unoriented. But, if $\Gamma$ is an even graph, i.e., if every vertex has even valency, then one can specify an orientation for $\widetilde{L}$ in such a way that the boundary of a shaded domain is an oriented circuit. Such an oriented diagram $\widetilde{L}$ is called a special diagram. In fact, every oriented link has a diagram which is special [B-Z, Theorem, p. 220].

We will use a special diagram $\widetilde{L}$ of a 2 -bridge link in order to evaluate $A_{L}$ and $B_{L}$.

Let $L$ be a 2-bridge link of type $(\alpha, \beta)$. Since we are only concerned about the braid index of $L$, we may assume w.l.o.g. that $0<\beta<\alpha$ and $\beta$ is odd. 


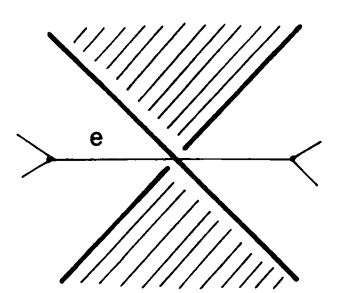

$w(e)=+1$

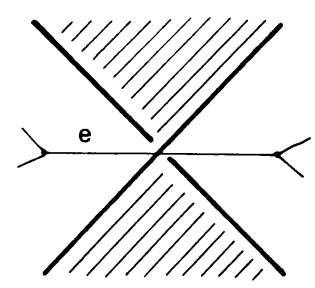

$w(e)=-1$

FIGURE 2

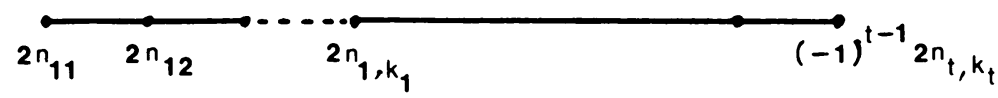

FIGURE 3

Then $\alpha /(\alpha-\beta)$ has a unique continued fraction form,

$$
\begin{aligned}
& {\left[2 n_{11}, \ldots, 2 n_{1, k_{1}},-2 n_{2,1}, \ldots,-2 n_{2, k_{2}}, \ldots,\right.} \\
& \left.(-1)^{t-1} 2 n_{t, 1}, \ldots,(-1)^{t-1} 2 n_{t, k_{t}}\right],
\end{aligned}
$$

where $n_{i, j}>0$ for any $i$ and $j$. Using this continued fraction from, we will construct a special diagram $\widetilde{L}$ of $L$. We should note that if $t>1$ then $\widetilde{L}$ is not alternating.

Now, since a 2-bridge link is an algebraic link (or an arborescent link), a weighted tree $T$ in $S^{2}$ will be associated with $L$. And $L$ is the boundary of a surface $F$ constructed by plumbing as specified by this weighted tree $T$. For a 2-bridge link $L, T$ is an arc-tree, i.e., $T$ has only two stumps. A stump is a vertex with valency 1 . See Figure 3.

The weight at the left-end stump is $2 n_{1,1}$.

Next we will define a weight $w(e)=+1$ or -1 to each edge $e$ on $T$ depending on whether the weight of the left-end of $e$ is positive or negative.

Example. 2-bridge link of type $(140,61)$. The continued fraction form of $\frac{140}{79}$ is $[2,4,-2,2,4]$.

Now take a new vertex $v_{0}$ off the tree $T$ and join $v_{0}$ to each vertex $v_{i, j}$ (which has weight $(-1)^{i-1} 2 n_{i, j}$ ) by disjoint simple arcs. The weight of a new edge is +1 or -1 according to whether $(-1)^{i-1} 2 n_{i, j}>0$ or not. The number, $s_{i, j}$, of edges joining $v_{0}$ and $v_{i, j}$ is determined by the following formulas.

(I) If $t=1$ and $k_{1}=1$, then $s_{11}=2 n_{11}$.

(II) Suppose $t \geq 2$ or $k_{1} \geq 2$, then

(1) $s_{11}=2 n_{11}-1$.

(2) Let $(i, j) \neq(1,1)$ or $\left(t, k_{t}\right)$. 


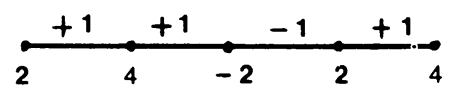

FIGURE 4

(i) If $v_{i, j}$ is the common end of two edges having the same weight, then $s_{i, j}=2 n_{i, j}-2$.

(ii) If $v_{i, j}$ is the common end of two edges having different weights, then $s_{i, j}=2 n_{i, j}$.

$$
\begin{aligned}
s_{t, k_{t}}=2 n_{t, k_{t}}-1 & \text { if } k_{t} \geq 2, \text { and } \\
s_{t, k_{t}}=2 n_{t, k_{t}}+1 & \text { if } k_{t}=1
\end{aligned}
$$

Now the resulting weighted graph $\Gamma$ (ignoring the weights of vertices) is what we wish to use as the graph of a (special) diagram of $L$. See Figure 5.

Since $\Gamma$ is an even graph, one can construct an oriented link diagram $\tilde{L}$ of some link $L$ such that $\Gamma$ is the graph of $\widetilde{L}$. $\widetilde{L}$ is a special diagram of a 2-bridge link $L$ of type $(\alpha, \beta)$. From our construction, we easily see

\section{Proposition 3.1.}

(1) For every vertex $v_{i, j}$ (except for $\left.v_{0}\right)$, the weight of $v_{i, j}\left(=(-1)^{i-1} 2 n_{i, j}\right.$ ) is equal to the sum of all weights of edges incident to $v_{i, j}$.

(2) $s_{i, j} \equiv 0 \quad(\bmod 2)$ for $(i, j) \neq(1,1)$ or $\left(t, k_{t}\right)$ and $s_{11} \equiv s_{t, k_{t}} \equiv 1 \quad(\bmod 2)$.

Using the special diagram $\widetilde{L}$ of a 2-bridge link $L$, we will determine $A_{L}$ and $B_{L}$.

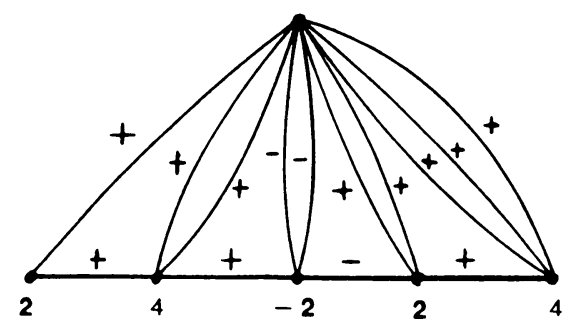

FIGURE 5 


\section{Proposition 3.2.}

$$
\begin{aligned}
& \text { (1) } a_{m}(L)=\sum_{i=1}^{t} k_{t}-2\left[\frac{t}{2}\right] \\
& \text { (2) } a_{l}(L)=-\sum_{i=0(2)} \sum_{j=1}^{k_{i}} 2 n_{i, j}-2\left[\frac{t}{2}\right]+\sum_{i=1}^{t} k_{i} . \\
& \text { (3) } b_{m}(L)=\sum_{i=1}^{t} k_{i}-2\left[\frac{t+1}{2}\right] . \\
& \text { (4) } b_{l}(L)=\sum_{i \equiv 1(2)} \sum_{j=1}^{k_{i}} 2 n_{i, j}+2\left[\frac{t+1}{2}\right]-\sum_{i=1}^{t} k_{i} . \\
& \text { (5) }\left|a_{L}\right|=\left|b_{L}\right|=1 . \\
& \text { (6) } \frac{1}{2}\left\{b_{l}(L)-a_{l}(L)\right\}=\sum_{i=1}^{t} \sum_{j=1}^{k_{i}}\left(n_{i, j}-1\right)+t .
\end{aligned}
$$

Proof. Since (6) follows from (1)-(5), we will prove only (1)-(5). A proof is an easy induction on $t$ and $k_{i}$. Thus we will omit most of our computations. In fact, if we apply a crossing change $\lambda^{\lambda} \rightarrow \searrow$ or $\searrow_{\searrow} \rightarrow \lambda^{\star}$ and splitting $X \rightarrow \asymp$ on a crossing on $\widetilde{L}$, the resulting link is also a 2-bridge link with a shorter continued fraction form. Therefore, we can apply the induction assumption on the resulting links. Then the formula (2.2) will prove the proposition

Case 1. $t=1$. If $k_{1}=1$, then $L$ is a torus link of type $\left(2 n_{1,1}, 2\right)$ and, hence, $A_{L}=-m l$ and $B_{L}=(-1)^{n_{11}-1} m^{-1} l^{2 n_{11}+1}$, which proves (3.2).

If $k_{1}>1$, apply a crossing change and splitting on the crossing on the leftmost edge joining $v_{0}$ and $v_{11}$. Then the link $L_{-}$and $L_{0}$ have, respectively, the following continued fraction forms:

$$
\begin{aligned}
L_{-}: & {\left[2 n_{13}, 2 n_{14}, \ldots, 2 n_{1, k_{1}}\right] \text { if } n_{11}=1, } \\
& {\left[2\left(n_{11}-1\right), 2 n_{12}, \ldots, 2 n_{1, k_{1}}\right] \text { if } n_{11} \geq 1 ; } \\
L_{0}: & {\left[2 n_{12}, 2 n_{13}, \ldots, 2 n_{1, k_{1}}\right] . }
\end{aligned}
$$

Since $-P_{L}(l, m)=l^{2} P_{L_{-}}(l, m)+l m P_{L_{0}}(l, m)$, a simple computation shows:

(1) If $n_{11}=1$, then $A_{L}=-m l A_{L_{0}}= \pm m^{k_{1}} l^{k_{1}}$ and

$$
B_{L}=-\operatorname{lm} B_{L_{0}}= \pm m^{k_{1}-2} l^{N_{1}-k_{1}+2},
$$

(2) If $n_{11} \geq 2$, then $A_{L}=-\operatorname{lm} A_{L_{0}}= \pm m^{k_{1}} l^{k_{1}}$ and

$$
B_{L}=-l^{2} B_{L_{-}}= \pm m^{k_{1}-2} l^{N_{1}-k_{1}+2}
$$

where $N_{1}=2 n_{11}+\cdots+2 n_{1, k_{1}}$. Now (3.3) proves (3.2) for the case $t=1$.

Case 2. $t \geq 2$ and $t \equiv 0(\bmod 2)$. 
We apply a crossing change and splitting on the crossing on the rightmost edge $e$ joining $v_{0}$ and $v_{t, k_{t}}$. We state only the continued fraction forms for $L_{+}$and $L_{0}$. The rest of the proof consists of straightforward computations using the induction assumptions.

(1) Suppose $k_{t}=1$. Then

$$
\begin{gathered}
L_{+}:\left[2 n_{11}, \ldots, 2 n_{1, k_{1}}, \ldots,(-1)^{t-2} 2 n_{t-1,1}, \ldots,(-1)^{t-2} \cdot 2 n_{t-1, k_{t-1}-1}\right], \\
\text { if } n_{t, 1}=1, \\
{\left[2 n_{11}, \ldots, 2 n_{1, k_{1}}, \ldots,(-1)^{t-2} 2 n_{t-1,1}, \ldots,\right.} \\
\left.(-1)^{t-2} 2 n_{t-1, k_{t-1}},(-1)^{t-1} 2\left(n_{t, 1}-1\right)\right], \\
\text { if } n_{t, 1} \geq 2 ; \\
L_{0}:\left[2 n_{11}, \ldots, 2 n_{1, k_{1}}, \ldots,(-1)^{t-2} 2 n_{t-1,1}, \ldots,(-1)^{t-2} 2 n_{t-1, k_{t-1}}\right],
\end{gathered}
$$

and

$$
A_{L}=-l^{-2} A_{L_{+}} \quad \text { and } \quad B_{L}=-l^{-1} m B_{L_{0}} .
$$

(2) Suppose $k_{t} \geq 2$. Then

$$
\begin{gathered}
L_{+}:\left[2 n_{11}, \ldots, 2_{n_{1}, k_{1}}, \ldots,(-1)^{t-1} 2 n_{t, 1}, \ldots,(-1)^{t-1} 2 n_{t, k_{t}-2}\right], \quad \text { if } n_{t, k_{t}}=1, \\
{\left[2 n_{11}, \ldots, 2 n_{1, k_{1}}, \ldots,(-1)^{t-1} 2 n_{t, 1}, \ldots,(-1)^{t-1} 2\left(n_{t, k_{t}}-1\right)\right], \quad \text { if } n_{t, k_{t}} \geq 2 ;} \\
L_{0}:\left[2 n_{1,1}, \ldots, 2 n_{1, k_{1}}, \ldots,(-1)^{t-1} 2 n_{t, 1}, \ldots,(-1)^{t} 2 n_{t, k_{t}-1}\right] .
\end{gathered}
$$

If $n_{t, k_{t}}=1$, then $A_{L}=-l^{-1} m A_{L_{0}}$ and $B_{L}=-l^{-1} m B_{L_{0}}$. If $n_{t, k_{t}} \geq 2$, then $A_{L}=-l^{2} A_{L_{+}}$and $B_{L}=-l^{-1} m B_{L_{0}}$.

Case 3. $t \geq 2$ and $t \equiv 1(\bmod 2)$. Since a proof is analogous to Case 2 , a proof will be omitted.

A proof of Proposition 3.2 is now complete.

\section{Proof of Theorem B(II)}

By Propositions 2.3 and 3.2(6), Theorem B will follow from Proposition 4.1 below.

Proposition 4.1. There is a diagram $\tilde{L}$ of a 2-bridge link $L$ which contains exactly $\sum_{i=1}^{t} \sum_{j=1}^{k_{i}}\left(n_{i j}-1\right)+t+1$ Seifert circles.

Proof. Let $\widetilde{L}$ be the diagram of $L$ constructed in $\S 3$. Since $\widetilde{L}$ is a special diagram, the number $s(\widetilde{L})$ of Seifert circles in $\widetilde{L}$ is exactly the number of domains $S^{2}-\Gamma(\widetilde{L})$. Let $V$ and $E$ denote, respectively, the number of vertices 
and edges in $\Gamma$. Then we see easily that

$$
\begin{aligned}
& \text { (1) } V=\sum_{i=1}^{t} k_{i}+1 \\
& \text { (2) } E=\sum_{i=1}^{t} \sum_{j=1}^{k_{i}}\left(2 n_{i j}-2\right)+\sum_{i=1}^{t} k_{i}+2 t-1 .
\end{aligned}
$$

Therefore, Euler's formula yields $s(\tilde{L})=E-V+2=2\left\{\sum_{i=1}^{t} \sum_{j=1}^{k_{i}}\left(n_{i j}-1\right)+t\right\}$. By comparing $s(\tilde{L})$ with $l$-span $P_{L}(l, m)=2\left\{\sum_{i=1}^{t} \sum_{j=1}^{k_{i}}\left(n_{i j}-1\right)+t\right\}$, we must eliminate $\sum_{i=1}^{t} \sum_{j=1}^{k_{i}}\left(n_{i j}-1\right)+t-1$ Seifert circles from $\widetilde{L}$.

Let $s_{i j}$ be the number of edges joining $v_{i j}$ and $v_{0}$ defined in (3.1). Then we can eliminate $\left[s_{i j} / 2\right]$ Seifert circles by simple deformation depicted in Figure 6 (which replace short arcs $\overparen{P_{i} P_{i}^{\prime}}$ by long arcs).

Repeated applications of this deformation eventually eliminate $\sum_{i, j}\left[s_{i j} / 2\right]=$ $\sum_{i, j}\left(n_{i j}-1\right)+t-1$ Seifert circles. This proves Proposition 4.1 and hence Theorem B. Q.E.D.

Theorem $\mathrm{B}$ and Theorem 1 in $[\mathrm{Y}]$ show that the exponent sum $\mathbf{e}(L)$ in a minimal braid representation of a 2-bridge link $L$ is equal to the writhe $w(\widetilde{L})$ $\left(=c_{+}(\widetilde{L})-c_{-}(\widetilde{L})\right)$ of a diagram $\widetilde{L}$ with a minimal number of Seifert circles, where $c_{+}(\widetilde{L})$ and $c_{-}(\tilde{L})$ denote, respectively, the number of positive and negative crossings in $\widetilde{L}$. Therefore, it is easy to determine $\mathbf{e}(L)$ for a 2-bridge link.

Theorem 4.2. Let $L$ be a 2-bridge link of type $(\alpha, \beta)$. Then

$$
\mathbf{e}(L)=\sum_{i \equiv 1(2)} \sum_{j=1}^{k_{i}} n_{i j}-\sum_{i \equiv 0(2)} \sum_{j=1}^{k_{i}} n_{i j}+\frac{1-(-1)^{t}}{2} .
$$

Proof. A diagram of $L$ with a minimal number of Seifert circles is obtained from a special diagram $\widetilde{L}$ by applying isotopic deformations shown in Figure 6 .

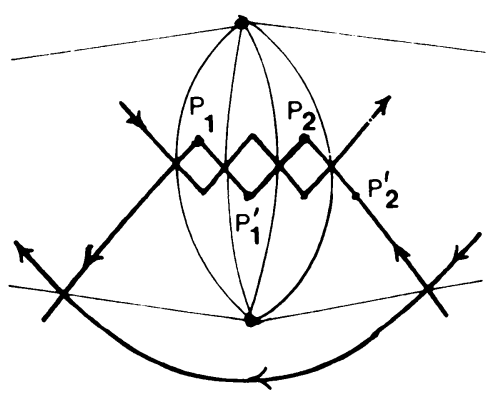

(a)

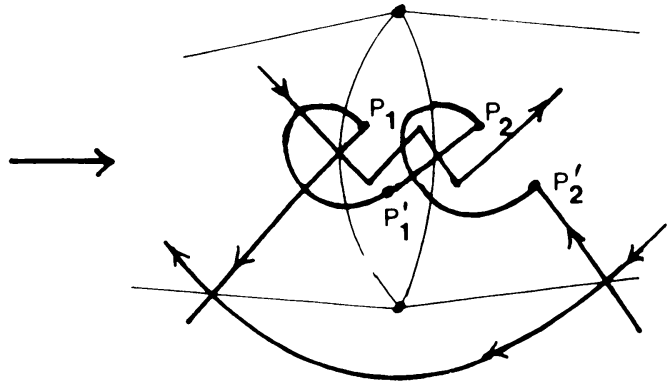

(b)

FigURE 6 
If $\overparen{P_{i} P_{i}^{\prime}}$ is an over-passing arc, then this deformation decreases $w(\tilde{L})$ by one. On the other hand, if $\overparen{P_{i} P_{i}^{\prime}}$ is an under-passing arc, then it increases $w(\widetilde{L})$ by one. Since the original diagram $\widetilde{L}$ has exactly $\sum_{i \equiv 1(2)}\left\{\sum_{j=1}^{k_{i}}\left(2 n_{i j}-2\right)+k_{i}+2\right\}-1$ positive crossings and $\sum_{i \equiv 0(2)}\left\{\sum_{j=1}^{k_{i}}\left(2 n_{i j}-2\right)+k_{i}+2\right\}$ negative crossings, the final diagram $\widehat{L}$ we obtained in Proposition 4.1 has the writhe

$$
\begin{aligned}
w(\widehat{L})= & \sum_{i \equiv 1(2)}\left\{\sum_{j=1}^{k_{i}}\left(2 n_{i j}-2\right)+k_{i}+2\right\} \\
& -1-\left\{\sum_{i \equiv 1(2)} \sum_{j=1}^{k_{i}}\left(n_{i j}-1\right)+\left[\frac{t-1}{2}\right]\right\} \\
& -\sum_{i \equiv 0(2)}\left\{\sum_{j=1}^{k_{i}}\left(2 n_{i j}-2\right)+k_{i}+2\right\}+\sum_{i \equiv 0(2)} \sum_{j=1}^{k_{i}}\left(n_{i j}-1\right)+\left[\frac{t}{2}\right] \\
= & \sum_{i \equiv 1(2)} \sum_{j=1}^{k_{i}} n_{i j}+2\left[\frac{t+1}{2}\right]-1-\left[\frac{t-1}{2}\right]-\sum_{i \equiv 0(2)} \sum_{j=1}^{k_{i}} n_{i j}-\left[\frac{t}{2}\right] .
\end{aligned}
$$

Since $2\left[\frac{t+1}{2}\right]-\left[\frac{t}{2}\right]-\left[\frac{t-1}{2}\right]-1=\left(1-(-1)^{t} / 2\right)$, we obtain (4.2).

The braid index of an alternating Montesinos link $L$ is also determined without difficulty. In fact, $L$ is an algebraic link which is associated with a weighted tree $T$. We state the following theorem without a proof.

Theorem 4.3. Let $T$ be a weighted tree associated with a Montensinos link $L$. Suppose that $T$ is positive and excessive. In other words, for each vertex $v$ of $T$, the weight $w(v)$ is positive and $w(v)$ is at least equal to the valency, $\operatorname{val}(v)$ at $v$. Then

$$
\mathbf{b}(L)=\sum\left[\frac{w(v)-\operatorname{val}(v)}{2}\right]+s,
$$

where the summation runs over all vertices in $T$ and $s$ denotes the number of stumps in $T$.

Remark 4.1. A link in Theorem 4.3 is a special alternating link. The braid index of an alternating Montesinos link associated with a excessive but not necessarily positive tree is also determined without much difficulty. However, the braid index of a general alternating algebraic link is not easy to determine, although some appropriate algorithm may exist. We do not pursue this problem any further in this paper. Rather, we will return to a proof of Theorem A in the next section.

\section{Proof of Theorem A(I)}

A proof of Theorem A is much more complicated than that of Theorem B. Contrary to a 2-bridge link, it is unlikely that an alternating fibered link has 
a neat diagram which reduces to a simpler alternating fibered link after one crossing change. Therefore, we must use a "global" argument, instead of a local one.

First we recall the definition of the ${ }^{*}$-product of two links. Let $L$ be an oriented link and $\widetilde{L}$ its diagram in $S^{2}$. A Seifert circle $C$ in $\widetilde{L}$ is classified as type (I) or type (II). $C$ is said to be of type (II) if both the interior and the exterior of $C$ contain other Seifert circles. Otherwise $C$ is of type (I). If there are no Seifert circles of type (II), the diagram is special. Suppose that there is a Seifert circle $C$ of type (II) in $\widetilde{L}$. If we erase the interior of $C$, we obtain a new link diagram of some link, say $L_{1}$. See Figure 7.

Example. $\tilde{L}$ has two Seifert circles $C$ and $C^{\prime}$, and $L$ is a ${ }^{*}$-product of three links $L_{1}, L_{2}$ and $L_{3}$, i.e., $L=L_{1} * L_{2} * L_{3}$.

Similarly, by erasing the part of $\widetilde{L}$ which is exterior to $C$, we will obtain the other link diagram $\widetilde{L}_{2}$ of a link $L_{2}$. We say then that the link $L$ is a (planar) "-product (or a (planar) Murasugi sum) of two links $L_{1}$ and $L_{2}$, written as $L_{1} * L_{2}$. Also the diagram $\widetilde{L}$ is called a (planar) * -product of two diagrams $\widetilde{L}_{1}$ and $\widetilde{L}_{2}$, written as $\widetilde{L}=\widetilde{L}_{1} * \widetilde{L}_{2}$. We can repeat the same argument as long as a diagram has Seifert circles of type (II) and $L$ will be decomposed into a finite number of links $L_{1}, L_{2}, \ldots, L_{k}$ with special diagrams $\widetilde{L}_{1}, \widetilde{L}_{2}, \ldots \widetilde{L}_{k}$. We write $L=L_{1} * L_{2} * \cdots * L_{n}$ and call it a (planar) ${ }^{*}$-products representation of $L$. Similarly, a diagram $\widetilde{L}$ is written as $\widetilde{L}=\widetilde{L}_{1} * \widetilde{L}_{2} * \cdots * \widetilde{L}_{k}$. (For more details, see [Mu2 or G].) Since the connected sum of two links can be considered a planar ${ }^{*}$-product of these links, we may assume that $L_{i}$ is prime. A *-product representation of a link is not unique. Nevertheless, this concept is particularly important in the study of alternating links. In fact, if $L$ is an alternating link, each component $L_{i}$ of a (planar) ${ }^{*}$-product representation of $L$ is a special alternating (either positive or negative) link.

Now suppose that $L$ is an alternating fibered link. Since a special alternating prime fibered link is an elementary torus link [Mu1], $L$ is a (planar) ${ }^{*}$-product of elementary torus links, i.e., torus links of type $\left(q_{i}, 2\right)$ or $\left(q_{i},-2\right)$, where $q_{i} \geq 2$.

Recall $c_{+}(\widetilde{L})$ and $c_{-}(\widetilde{L})$ to denote the number of positive and negative crossings in $\widetilde{L}$. Therefore, $c(\widetilde{L})=c_{+}(\widetilde{L})+c_{-}(\widetilde{L})$. Write $w(\widetilde{L})=c_{+}(\widetilde{L})-c_{-}(\widetilde{L})$ and $s(\widetilde{L})$ is the number of Seifert circles in $\widetilde{L} . h_{+}(\widetilde{L})$ and $h_{-}(\widetilde{L})$ denote the number of positive and negative ${ }^{*}$-components in $\widetilde{L}$, and hence $h(\widetilde{L})=$ $h_{+}(\widetilde{L})+h_{-}(\widetilde{L})$. Then Theorem A will follow from the following

Proposition 5.1. If $L$ is an alternating fibered link, then

$$
\begin{aligned}
& \text { (1) } A_{L}=(-1)^{c+h_{+}} m^{c-h-2 h}-l^{w-h}, \\
& \text { (2) } B_{L}=(-1)^{c+h} m^{c-h-2 h_{+}} l^{w+h},
\end{aligned}
$$

where, for simplicity, we delete $\widetilde{L}$ from the notation. 


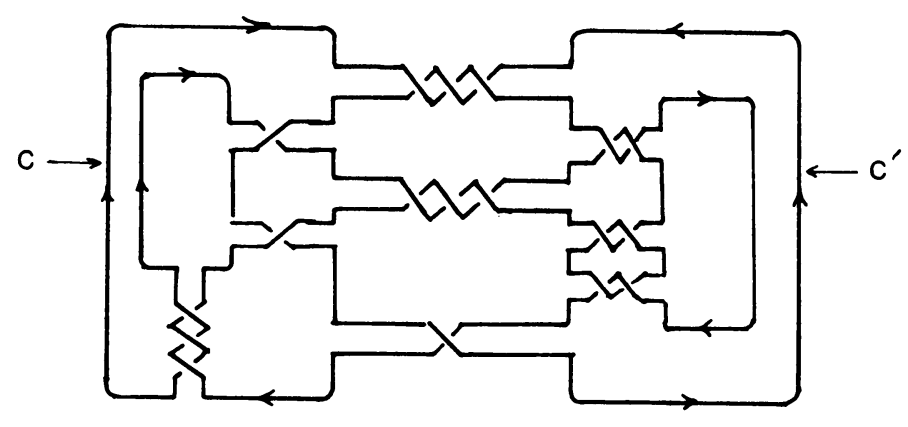

L

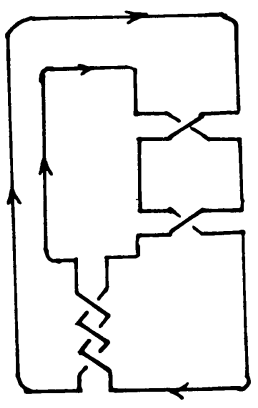

$L_{1}$

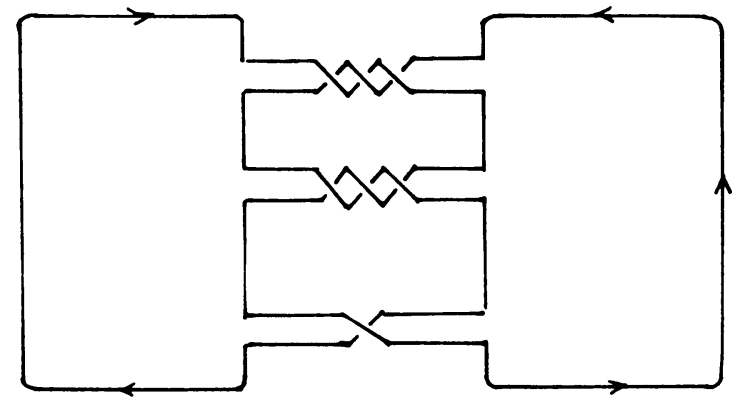

$L_{2}$

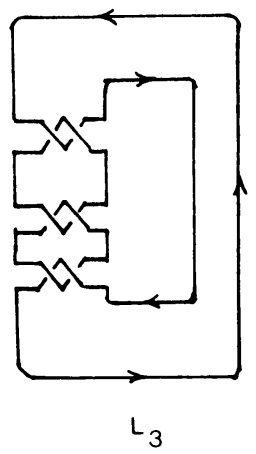

FIGURE 7

In fact, since $s(\widetilde{L})=h(\widetilde{L})+1$, we see that $l$-span $P_{L}(l, m)=2 h(\widetilde{L})=$ $2\{s(\widetilde{L})-1\}$ and hence, $\mathbf{b}(L)=s(\widetilde{L})=h(\widetilde{L})+1$ by Proposition 2.3. This proves Theorem A.

Now, for the mirror image $L^{*}$ of $L$, we know that $P_{L^{*}}(l, m)=P_{L}\left(l^{-1}, m\right)$ [L-M] and hence $A_{L}$ is obtained immediately from $B_{L^{*}}$. Therefore, we need only prove $(5.1)(2)$. 
A proof of $(5.1)(2)$ will be given by induction on $h(\tilde{L})$ and $c(\tilde{L})$.

Suppose $h(\widetilde{L})=1$. Then we may assume that $h_{+}(\widetilde{L})=1$ and $h_{-}(\tilde{L})=0 . L$ is a torus link of type $(q, 2), q \geq 2$. Then a simple computation [M-L] shows that $B_{L}=(-1)^{q} m^{q-3} l^{q+1}$. Since $c(\widetilde{L})=q, h_{+}(\widetilde{L})=1=h(\widetilde{L}), h_{-}(\widetilde{L})=0$ and $w(\widetilde{L})=q,(5.1)(2)$ follows immediately.

Now suppose $h \geq 2$. Suppose furthermore that all ${ }^{*}$-components are positive. Then $h(\widetilde{L})=h_{+}(\tilde{L})$ and $h_{-}(\widetilde{L})=0$. Since $\widetilde{L}$ is an alternating diagram, $L$ must be a connected sum of these positive elementary torus links $L_{i}$. (We need the assumption here that $L$ be alternating.) Then it is known [L-M] that $B_{L}=\prod_{i=1}^{h} B_{L_{i}}$, and hence we obtain (5.1)(2).

Therefore, we may assume in the rest of this section and the next that

$$
h_{+}(\widetilde{L}) \neq 0 \neq h_{-}(\widetilde{L}) \text {. }
$$

Now, given a link $L$ and its diagram $\widetilde{L}$, there are, say, $k$ crossings $c_{1}$, $c_{2}, \ldots, c_{k}$ on $\tilde{L}$ such that crossing changes at these crossings make $L$ a trivial link $L_{0}$. Then the polynomial $P_{L}(l, m)$ of $L$ is given by the formula [Mu3].

$$
\begin{aligned}
P_{L}(l, m)= & (-1)^{k} l^{2(y-x)} P_{L_{0}}(l, m) \\
& +(-1) \sum_{d=1}^{k} m^{d} \sum_{1 \leq j_{1}<\cdots<j_{d} \leq k} l^{d-2 u_{d}} P_{L_{j_{1} \cdots j_{d}}}(l, m),
\end{aligned}
$$

where $y$ and $x$ are, respectively, the number of positive and negative crossings among $\left\{c_{1}, \ldots, c_{k}\right\}, u_{d}$ is the number of negative crossings among $\left\{c_{j_{i}}, \ldots\right.$, $\left.c_{j_{d}}\right\}$, and $L_{j_{1} j_{2} \cdots j_{d}}$ is a link diagram obtained from $\widetilde{L}$ by splitting each of crossings $c_{j_{1}}, \ldots, c_{j_{d}}$. We can choose these $k$ crossings so that each link diagram $L_{j_{1} \cdots j_{d}}$ is connected. If $L$ is an alternating fibered link, then, to each $\left\{j_{1}, \ldots, j_{d}\right\}, L_{j_{1} \cdots j_{d}}$ is an alternating fibered link diagram or a trivial knot. It cannot be a trivial link, since $L_{j_{1} \cdots j_{d}}$ is alternating and connected. We should note that $L_{j_{1} \cdots j_{d}}$ may not be reduced, i.e., it may contain removable (or nugatory) crossings.

Now, to prove $(5.1)(2)$, we carefully choose a set of crossings on which crossings changes and splittings are applied.

Let $\widetilde{L}$ be ${ }^{*}$-product of elementary torus link diagrams $\widetilde{L}_{i}$ of type $\left(q_{i}, 2\right)$ or $\left(q_{i},-2\right), q_{i} \geq 2$.

Lemma 5.2. There are, say, $k$ crossings $c_{1}, c_{2}, \ldots, c_{k}$ such that (1) crossing changes at $c_{1}, c_{2}, \ldots, c_{k}$ make $\tilde{L}$ a trivial link, and (2) for each $i, i=$ $1,2, \ldots, h$, the number $\alpha_{i}$ of crossing changes applied on $\widetilde{L}_{i}$ is at least one but at most $q_{i}-1$, i.e., $0<\alpha_{i}<q_{i}$. In other words, each $\widetilde{L}_{i}$ has at least one crossing in $\left\{c_{1}, c_{2}, \ldots, c_{k}\right\}$, but it never has all crossings in $\left\{c_{1}, c_{2}, \ldots, c_{k}\right\}$. 
Note that if $\alpha_{i}=q_{i}-1$, then a diagram of some $L_{j_{1} \cdots j_{d}}$ is not reduced. Also we may not need to apply crossing changes at all crossings $c_{1}, \ldots, c_{k}$ to make $\widetilde{L}$ trivial. In other words, we apply some unnecessary crossing changes to $L$ to prove Lemma 5.2. We postpone a proof of Lemma 5.2 to the Appendix.

Now, we proceed to a proof of Proposition 5.1. Let $\left\{c_{1}, c_{2}, \ldots, c_{k}\right\}$ be a set of crossings found in Lemma 5.2. Consider (5.3). Write $C=l^{2(y-x)} P_{L_{0}}(l, m)$ and $f(l, m)=\sum_{d=1}^{k} m^{d} \sum_{1 \leq j_{1}<\cdots<j_{d} \leq k} l^{d-2 u_{d}} P_{L_{j_{1} \cdots j_{d}}}(l, m)$. Then we claim

\section{Proposition 5.3.}

$$
B_{f}=(-1)^{c+h} m^{c-h-2 h_{+}} l^{w+h} \text {. }
$$

A proof of Proposition 5.3 will be given in the next section. In the rest of this section, we will prove, using $(5.4)$, that $B_{f}$ is in fact the maximum term of $P_{L}(l, m)$.

Proposition 5.4. $B_{f}=B_{L}$. In other words, $B_{C}$ cannot be the maximum term of $P_{L}(l, m)$.

Proof. Let $\mu$ be the number of components in $L$. Since $L_{0}$ is a trivial $\mu$ component link,

$$
P_{L_{0}}(l, m)=\left(-\left(l+l^{-1}\right) / m\right)^{\mu-1}
$$

and hence

$$
C=l^{2(y-x)}\left(-\left(l+l^{-1}\right) / m\right)^{\mu-1} .
$$

Therefore, we have

$$
B_{C}=(-1)^{\mu-1} m^{-(\mu-1)} l^{2(y-x)+(\mu-1)} .
$$

Now we may assume w.l.o.g. that $2 y \leq c_{+}(\tilde{L})$. (Otherwise consider "complementary" crossing changes. That is, if we apply only crossing changes at those crossings which are not in the set $\left\{c_{1}, c_{2}, \ldots, c_{k}\right\}$, then we have another set of crossings that satisfies all conditions in Lemma 5.2. For this set, it holds that $2 y \leq c_{+}(\widetilde{L})$.)

Suppose that $B_{C}$ is the maximum term of $P_{L}(l, m)$. Then $2(y-x)+\mu-$ $1 \geq w(\widetilde{L})+h$. But since the maximal degree of $P_{L}(l, m)$ w.r.t. $l$ is at most $w(\widetilde{L})+s(\widetilde{L})-1=w(\widetilde{L})+h[\mathrm{Mo}]$, we must have

$$
2(y-x)+\mu-1=w(\widetilde{L})+h .
$$

Furthermore, since $-(\mu-1)$ is the minimal degree of $P_{L}(l, m)$ w.r.t. $m$ [L-M], we see

$$
-(\mu-1)=c(\widetilde{L})-h-2 h_{+} .
$$

Combining (5.6) and (5.7), we obtain that $2(y-x)=2 c_{+}(\widetilde{L})-2 h_{+}$. This is impossible, however, since $2 c_{+}(\widetilde{L}) \geq 2 h_{+}+2 y$ and $x>0$, proving Proposition 5.4 . 


\section{Proof of Theorem A(II)}

This section will be devoted to the proof of Proposition 5.3.

Suppose that some $L_{j_{1}, \ldots, j_{d}}$ is not reduced. Let $L_{j_{1}, \ldots, j_{d}}=\widehat{L}_{1} * \widehat{L}_{2} * \cdots *$ $\widehat{L}_{h}$. Since $L_{j_{1}, \ldots, j_{d}}$ is not reduced, some of $\widehat{L}_{j}$ represents trivial knots, i.e., all crossings but one of $\widehat{L}_{j}$ must be split. For simplicity, each of $\widehat{L}_{1} \cdots \widehat{L}_{\lambda}$ is a trivial knot with one positive crossing and each of $\widehat{L}_{\lambda+1}, \ldots, \widehat{L}_{\lambda+\nu}$ is a trivial knot with one negative crossing. Then $L_{j_{1}, \ldots, j_{d}}$ is isotopic to a reduced alternating fibered link $\widehat{L}_{j_{1} \ldots j_{d}}=\widehat{L}_{\lambda+\nu+1} * \cdots * \widehat{L}_{h}$, and hence we have

$$
\begin{aligned}
& \text { (1) } c\left(\widehat{L}_{j_{1} \cdots j_{d}}\right)=c(\widetilde{L})-d-(\lambda+\nu), \\
& \text { (2) } h\left(\widehat{L}_{j_{1} \cdots j_{d}}\right)=h(L)-(\lambda+\nu), \\
& \text { (3) } h_{+}\left(\widehat{L}_{j_{1} \cdots j_{d}}\right)=h_{+}(L)-\lambda, \\
& \text { (4) } h_{-}\left(\widehat{L}_{j_{1} \cdots j_{d}}\right)=h_{-}(L)-\nu, \\
& \text { (5) } w\left(\widehat{L}_{j_{1} \cdots j_{d}}\right)=w(\widetilde{L})-d+2 u_{d}-\lambda+\nu .
\end{aligned}
$$

Since $\widehat{L}_{j_{1}, \ldots, j_{d}}$ is reduced, we can apply the induction assumption on $\widehat{L}_{j_{1}, \ldots, j_{d}}$ to show that

$$
B_{L_{j_{1} \cdots j_{d}}}=B_{\widehat{L}_{j_{1} \cdots j_{d}}}=(-1)^{c-d+h_{-}-\lambda} m^{c-h-2 h_{+}-d+2 \lambda} l^{w+h-d+2 u_{d}-2 \lambda},
$$

and hence we have

$$
m^{d} l^{d-2 u_{d}} B_{L_{j_{1} \cdots j_{d}}}=(-1)^{c-d+h_{-}-\lambda} m^{c-h-2 h_{+}+2 \lambda} l^{w+h-2 \lambda} .
$$

From (6.2), we see that if $\lambda \neq 0$ then $m^{d} l^{d-2 u_{d}} B_{L_{j_{1} \ldots j_{d}}}$ cannot be the maximum term of $f(l, m)$. Therefore, to prove (5.4), it is sufficient to show that $\sum_{d=1}^{k} \sum_{1 \leq j_{1}<\cdots<j_{d} \leq k}(-1)^{c-d+h_{-}}=(-1)^{c+h_{-}+1}$, or equivalently,

$$
\sum_{d=1}^{k} \sum_{1 \leq j_{1}<\cdots<j_{d} \leq k}(-1)^{d}=-1,
$$

where the summation is further restricted to $\left\{j_{1}, \ldots, j_{d}\right\}$ such that $L_{j_{1} \cdots j_{d}}$ has $\lambda=0$. Thus we need to count the number of links $L_{j_{1}, \ldots, j_{d}}$ with $\lambda=0$.

If $\lambda=0$ for all $L_{j_{1} \ldots j_{d}}$ or, in particular, if all $L_{j_{1}, \ldots, j_{d}}$ are reduced, then since there are exactly $\left(\begin{array}{l}d \\ k\end{array}\right)$ choices of sequences $j_{1}, \ldots, j_{d}$ from $k$ distinct indices, $1,2, \ldots, k$, we see

$$
\sum_{d=1}^{k} \sum_{1 \leq j_{1}<\cdots<j_{d} \leq k}(-1)^{d}=\sum_{d=1}^{k}\left(\begin{array}{l}
k \\
d
\end{array}\right)(-1)^{d}=-1
$$

and we are done. To prove (6.3) in general, let $\mathscr{S}=\left\{c_{1}, c_{2}, \ldots, c_{k}\right\}$ be crossings found in Lemma 5.2. Since $h_{+}(\widetilde{L}) \neq 0 \neq h_{-}(\widetilde{L})$, some crossings in 
are positive and some are negative. For simplicity, let $L_{1}, \ldots, L_{p}$ all be positive links $L_{i}$ with $\alpha_{i}=q_{i}-1, i=1,2, \ldots, p$, but each of the remaining $h-p$ links $L_{p+1}, \ldots, L_{h}$ has either negative crossings or has at most $q_{i}-2$ positive crossings in $\mathscr{S}$. Note from Lemma 5.2 that $0 \leq p<h$. Denote by $\xi$ the left-hand side of equation (6.3). $\xi$ will easily be evaluated as follows.

We see from (6.2) that $m^{d} l^{d-2 u_{d}} B_{L_{j_{1}} \ldots, j_{d}}$ is the maximum term of $f(l, m)$ if and only if, to each $i, l \leq i \leq p$, all $\alpha_{i}$ crossings of $L_{i}$ contained in $\mathscr{S}$ never occur in $\left\{c_{j_{1}}, \ldots, c_{j_{d}}\right\}$. The number $\xi_{d}$ of such links $L_{j_{1} \cdots j_{d}}$ is given by

$$
\xi_{d}=\sum_{i_{1}, \ldots, i_{p}} \sum_{m_{p+1} \cdots m_{h}}\left(\begin{array}{c}
\alpha_{1} \\
i_{1}
\end{array}\right) \cdots\left(\begin{array}{c}
\alpha_{p} \\
i_{p}
\end{array}\right)\left(\begin{array}{c}
\alpha_{p+1} \\
m_{p+1}
\end{array}\right) \cdots\left(\begin{array}{c}
\alpha_{h} \\
m_{h}
\end{array}\right),
$$

where the summation runs over all $i_{1}, \ldots, i_{p}, m_{p+1}, \ldots, m_{h}$ such that $0 \leq$ $i_{k} \leq \alpha_{k}-1, k=1,2, \ldots, p$ and $0 \leq m_{r} \leq \alpha_{r}, r=p+1, \ldots, h$. Putting

$$
\xi_{0}=\left(\begin{array}{c}
\alpha_{1} \\
0
\end{array}\right) \cdots\left(\begin{array}{c}
\alpha_{n} \\
0
\end{array}\right)=1 \text {, }
$$

we can write $\xi=\sum_{d=0}^{k}(-1)^{d} \xi_{d}-1$. However, a straightforward computation shows that

$$
\begin{aligned}
\sum_{d=0}^{k}(-1)^{d} \xi_{d}= & \left\{\sum(-1)^{i_{1}+\cdots+i_{p}}\left(\begin{array}{c}
\alpha_{1} \\
i_{1}
\end{array}\right) \cdots\left(\begin{array}{c}
\alpha_{p} \\
i_{p}
\end{array}\right)\right\} \\
& \times\left\{\sum(-1)^{m_{p+1}+\cdots+m_{h}}\left(\begin{array}{c}
\alpha_{p+1} \\
m_{p+1}
\end{array}\right) \cdots\left(\begin{array}{c}
\alpha_{h} \\
m_{h}
\end{array}\right)\right\} .
\end{aligned}
$$

Since $h-p>0$, the second factor is

$$
\sum(-1)^{m_{p+1}+\cdots+m_{k}}\left(\begin{array}{c}
\alpha_{p+1} \\
m_{p+1}
\end{array}\right) \cdots\left(\begin{array}{c}
\alpha_{h} \\
m_{h}
\end{array}\right)=\prod_{i=p+1}^{h} \sum_{m_{i}=0}^{\alpha_{i}}(-1)^{m_{i}}\left(\begin{array}{c}
\alpha_{i} \\
m_{i}
\end{array}\right)=0,
$$

and hence $\xi=-1$ as required. This proves Proposition 5.3, and the proof of Theorem $\mathrm{A}$ is now complete.

Theorem A immediately implies

Proposition 6.2. For an alternating fibered link $L, \mathbf{e}(L)=w(\widetilde{L})$, where $\widetilde{L}$ is a reduced alternating diagram of $L$.

\section{Corollaries}

We begin with a proof of Corollary 1.

Proof of Corollary 1. Since $h(\widetilde{L})=\mathbf{b}(L)-1, h(\widetilde{L})$ is an alternating-link invariant. Furthermore, it follows from Theorem 5.4 in [Mu2] that the signature

$$
\begin{aligned}
\sigma(L) & =-\left\{\left(c_{+}(\widetilde{L})-h_{+}(\widetilde{L})\right)-\left(c_{-}(\widetilde{L})-h_{-}(\widetilde{L})\right)\right\} \\
& =-\left\{c_{+}(\widetilde{L})-c_{-}(\tilde{L})\right\}+h_{+}(\tilde{L})-h_{-}(\tilde{L}) .
\end{aligned}
$$


Since $c_{+}(\widetilde{L})-c_{-}(\widetilde{L})=w(\widetilde{L})$ is an alternating-link invariant [Mu5], so is $h_{+}(\widetilde{L})-$ $h_{-}(\widetilde{L})$. Now the invariances of $h_{+}(\widetilde{L})$ and $h_{-}(\widetilde{L})$ follow immediately.

Remark 7.1. The invariances of $h_{+}(\widetilde{L})$ and $h_{-}(\widetilde{L})$ are also proved by Traczyk [Tr]. He proves that if $L$ is a (nonsplit) alternating fibered link, then, for the homfly polynomial $Q_{L}(x, y, z)$ of $L$,

$$
\begin{aligned}
& d_{\min }^{x} Q_{L}(x, y, z)=-c_{+}(\widetilde{L})+h_{+}(\tilde{L}), \\
& d_{\min }^{y} Q_{L}(x, y, z)=-c_{-}(\widetilde{L})+h_{-}(\widetilde{L}),
\end{aligned}
$$

where $d_{\min }^{x} Q$ and $d_{\min }^{y} Q$ denote the minimal degree of $Q$ w.r.t. $x$ and $y$, respectively. Since $c_{+}(\widetilde{L})$ and $c_{-}(\widetilde{L})$ are alternating-link invariants [Mu6], so are $h_{+}(\widetilde{L})$ and $h_{-}(\widetilde{L})$.

Proof of Corollary 2. Let $L$ be an alternating fibered link and $\widetilde{L}$ its reduced alternating diagram. Then it is known [Mu2] that $\operatorname{deg} \Delta_{L}(t)=c(\widetilde{L})-s(\widetilde{L})+1$. Since $\widetilde{L}$ is reduced, it follows that $\mathbf{b}(L)=h+1=s(\widetilde{L})=c(\widetilde{L})-\operatorname{deg} \Delta_{L}(t)+$ 1. Q.E.D.

Next we show a few immediate consequences of the main theorems.

Proposition 7.1. Let $L_{i}, i=1,2$, be either an alternating fibered link or a 2-bridge link. Then

$$
\mathbf{b}\left(L_{1} \# L_{2}\right)=\mathbf{b}\left(L_{1}\right)+\mathbf{b}\left(L_{2}\right)-1 \text {. }
$$

This solves Problem 10 in [B] for these links. A proof follows from the fact that $P_{L}(l, m)$ is multiplicative w.r.t. a connected sum.

Proposition 7.2. Let $L$ be a 2-bridge link of type $(\alpha, \eta)$, where $0<\beta<\alpha$ and $\beta$ is odd. Then

$$
\begin{array}{ll}
\text { (1) } \quad \mathbf{b}(L)=2 \quad \text { iff } \beta=1 . \\
\text { (2) } \mathbf{b}(L)=3 \quad \text { iff either }
\end{array}
$$

(i) for some $p, q>0, \alpha=2 p q+3 p+3 q+4$ and $\beta=2 p+3$, or

(ii) for some $p>0, \alpha=2 p q+p+q+1$ and $\beta=2 p+1$.

Proof. A proof of (1) is obvious. To show (2), we note by Theorem B that $\mathbf{b}(L)=3$ iff the continued fraction form of $L$ is either (1) $t=1$ and all $n_{1 j}=1$ but one $n_{1 k}=2$, i.e. $[2,2, \ldots, 2,4,2, \ldots, 2]$, or (ii) $t=2$ and $n_{i j}=1$ for all $i$ and $j$, i.e., $[2,2, \ldots, 2,-2,-2, \ldots,-2]$. A simple computation shows that, for each case, $\alpha$ and $\beta$ have the form given in Proposition 7.2.

Remark 7.2. Proposition 7.2 proves the conjecture by Przytycki [P].

We call an $n$-braid $\gamma=\sigma_{i_{1}}^{\varepsilon_{1}} \cdots \sigma_{i_{k}}^{\varepsilon_{k}}, \varepsilon_{i}= \pm 1,1 \leq i_{1}, \ldots, i_{k} \leq n-1$, a homogeneous braid if $\varepsilon_{j}=\varepsilon_{l}$ whenever $i_{j}=i_{l} . \gamma$ is called an alternating braid if $\varepsilon_{j}=\varepsilon_{l}$ iff $i_{j} \equiv i_{l}(\bmod 2) . \gamma$ is called reduced if each $\sigma_{j}, 1 \leq j \leq n-1$, appears at least twice in $\gamma$. 
Proposition 7.3. If $\gamma$ is an alternating and reduced $n$-braid, then the braid index of the closure $\hat{\gamma}$ of $\gamma$ is exactly $n$.

Proof. $\gamma$ is an alternating fibered link with a natural downward orientation. Since $\gamma$ is reduced, $\hat{\gamma}$ has exactly $n$ (nontrivial) $*$-components, and hence Proposition 7.3 follows from Theorem A.

The closure of a homogeneous $n$-braid may have a smaller braid index than $n$, but if it has the braid index $n$, then we can determine the minimal number of crossings of its closure. In fact, let $\mathbf{c}(L)$ denote the minimal number of crossings among all diagrams that a link $L$ can have. Then we can prove

Proposition 7.4. Let $\gamma$ be a homogeneous $n$-braid. Let $d_{i}$ be the exponent sum of $\sigma_{i}$ in $\gamma$. If $\mathbf{b}(\hat{\gamma})=n$ or if $\gamma$ is a reduced pure $n$-braid, then $\mathbf{c}(\hat{\gamma})=\sum_{i=1}^{n-1}\left|d_{i}\right|$. Proof. If $\gamma$ is reduced and pure, then $\mathbf{b}(\hat{\gamma})=n$ by Proposition 2.2. Therefore, we assume that $\mathbf{b}(\hat{\gamma})=n$. Let $d=\sum_{i=1}^{n-1}\left|d_{i}\right|$ and let $\widetilde{L}$ be any diagram of $L$. Then the degree of the reduced Alexander polynomial $\Delta(t)$ of $\hat{\gamma}$ is bounded above by $c(\widetilde{L})-s(\widetilde{L})+1$. On the other hand, since $\gamma$ is homogeneous, $\operatorname{deg} \Delta(t)=c(\tilde{\gamma})-s(\tilde{\gamma})+1=d-n+1$, where $\tilde{\gamma}$ is the diagram obtained from $\hat{\gamma}$ as a closed $n$-braid. Therefore $c(\widetilde{L})-s(\widetilde{L})+1 \geq d-n+1$ and hence $c(\widetilde{L})-d \geq s(\widetilde{L})-n \geq 0$ by Proposition 2.1. Therefore $c(\widetilde{L}) \geq d$, i.e., $d=\mathbf{c}(\hat{\gamma})$.

As a typical application, we have

Proposition 7.5. Let $L(q, r)$ be the torus link of type $(q, r)$, where $2 \leq q \leq r$. Then $\mathbf{c}(L(q, r))=r(q-1)$.

Proof. The minimal bridge number of a link is at most equal to its braid index. Since the minimal bridge number of $L(q, r)$ is $q$ [S], it follows that $\mathbf{b}(L(q, r))=q$. (This fact will also follow from [J, Theorems 9.7 and 15.1] for a torus knot of type $(q, r)$.) Since $L(q, r)$ is represented as the closure of a homogeneous $q$-braid $\left(\sigma_{1} \sigma_{2} \cdots \sigma_{q-1}\right)^{r}$, the proposition follows immediately.

\section{Special alternating LinKs}

To determine the braid index of an alternating link $L$, we may need the braid index of each $*$-component of $L$, which is a special alternating link. Generally, there is no known algorithm which can be used to determine the braid index of a special alternating link. However, there are some particular types of special alternating links besides 2-bridge links and fibered links, whose braid indices are easily determined.

Let $L$ be a special alternating link and $\widetilde{L}$ a reduced special alternating diagram. Let $\Gamma(\widetilde{L})$ be the weighted graph associated with $\widetilde{L}$. Since $\widetilde{L}$ is special alternating, we may assume that $\Gamma$ is positive and even. A (planar) graph $\Gamma$ (or the link diagram $\widetilde{L}$ associated with $\Gamma$ ) is said to be of the nonmultiple type if no two vertices in the dual graph $\Gamma^{*}$ of $\Gamma$ are connected by a single edge. 


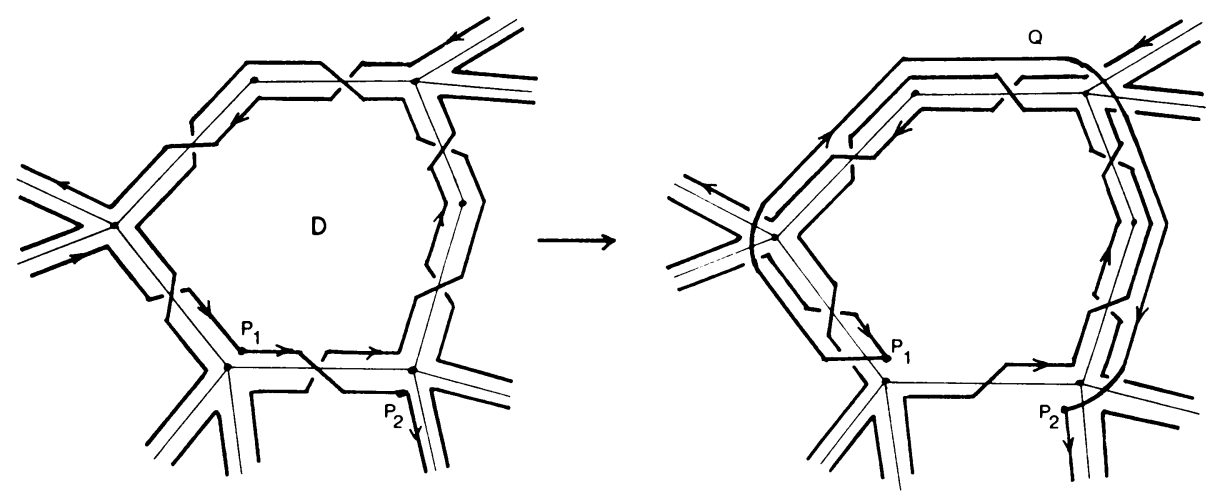

FIGURE 8

Theorem 8.1. Let $L$ be a special alternating link and $\tilde{L}$ a reduced special alternating diagram. Let $s(\widetilde{L})$ denote the number of Seifert circles in $\widetilde{L}$. Then $\mathbf{b}(L)=s(\widetilde{L})$ iff $\Gamma(\widetilde{L})$ is of the nonmultiple type.

The "if" part of the theorem was originally stated by K. Kobayashi in [K2]. However, we will prove both implications.

Proof of Theorem 8.1. First we prove the "only if" part. Suppose that $\Gamma(\widetilde{L})$ is not of the nonmultiple type. Then it suffices to prove that $L$ has a diagram $\widehat{L}$ such that $s(\widehat{L})<s(\widetilde{L})$. In fact, then, by Proposition 2.1, $\mathbf{b}(L) \leq s(\widehat{L})<s(\widetilde{L})$.

Since $\Gamma(\widetilde{L})$ is not of the nonmultiple type, there are two vertices in the dual graph $\Gamma^{*}$ of $\Gamma(\tilde{L})$ which are joined by a single edge. Therefore, there is an edge $e$ in $\Gamma$ which connects two nontwigs, say $v_{1}$ and $v_{2}$, where a twig is a vertex with valency two. Let $D$ be the domain in $S^{2}-\Gamma$ whose boundary contains $e . D$ corresponds to one shaded domain in $S^{2}-\widetilde{L}$. See Figure 8(a).

Take two points $P_{1}$ and $P_{2}$ on an overcrossing arc that passes over the crossing associated with $e$. Then replace the short arc $\overparen{P_{1} P_{2}}$ by the long path $\overparen{P_{1} Q P_{2}}$ depicted in Figure $8(\mathrm{~b}) . \overparen{P}_{P_{1} Q P_{2}}$ is an over-crossing arc which lies outside $D$ but is close to the boundary of $D$. It is easy to see from Figure 8(b) that a new diagram $\widehat{L}$ has $s(\widetilde{L})-1$ Seifert circles. This proves the "only if" part of Theorem 8.1. Therefore, if $\Gamma(\widetilde{L})$ is not of the nonmultiple type, then, by $(2.3)$,

$$
\text { l- } \operatorname{span} P_{L}(l, m)<2\{s(\tilde{L})-1\} .
$$

For the "if" part, suppose that $\Gamma(\tilde{L})$ is of the nonmultiple type. Then, by Proposition 2.3, it is enough to prove

$$
l \text {-span } P_{L}(l, m)=2\{s(\widetilde{L})-1\} .
$$

We evaluate $a_{l}(L)$ and $b_{l}(L) ; a_{l}(L)$ is easily evaluated even for positive links. 
Proposition 8.2. Let $L$ be a (nonsplit) positive link. Let $r=\operatorname{deg} \Delta_{L}(t)$. Then $A_{L}=(-1)^{r} m^{r} l^{r}$.

For a positive link $L$, it it is shown [K1] that $r=a_{l}(L)$ and $r$ is also the maximal degree of $m$ in $P_{L}(l, m)$. Since $L$ is a positive link, almost the same argument employed in [Mu3] can be used to prove Proposition 8.2 by induction on $c(\widetilde{L})$. What we need to prove (8.2) is the fact that $r=a_{l}(L)$, which is already proved in [K1], and hence we omit a proof. For details, see [K1] or [Mu3].

Now if $\widetilde{L}$ is reduced special alternating diagram of $L$, then $r=c(\widetilde{L})-$ $s(\widetilde{L})+1$, and hence (8.2) will follow from

$$
b_{l}(L)=c(\widetilde{L})+s(\widetilde{L})-1 .
$$

We will prove $(8.3)$ by induction on $c(\widetilde{L})$. We may assume w.l.o.g. that $\widetilde{L}$ is positive.

If $c(\widetilde{L})=2$, then $\Gamma$ is $\bigcirc$ and $(8.3)$ obviously holds.

Suppose that two nontwigs $v_{1}$ and $v_{2}$ are connected by a sequence of twigs $w_{1}, w_{2}, \ldots, w_{k}, k \geq 1$.

Let $e_{i}$ be an edge joining $w_{i}$ and $w_{i+1}, i=0,1, \ldots, k$, where $w_{0}=v_{1}$ and $w_{k+1}=v_{2}$. Apply the formula (2.2) on a crossing associated with the first edge $e_{1}$. For convenience, we denote the polynomials $P(l, m)$ of $L, L_{-}$and $L_{0}$ by $P_{k+1}, P_{k-1}$ and $P_{k}$, respectively, since these links contain exactly $k+1, k-1$ and $k$ edges connecting $v_{1}$ and $v_{2}$. Now (2.2) implies that $-P_{k+1}(l, m)=$ $\operatorname{lm} P_{k}(l, m)+l^{2} P_{k-1}(l, m)$. Repeated applications of (2.2) yield, for $k-n \geq 0$,

$$
P_{k+1}(l, m)=q_{n}(l, m) P_{k-n+1}(l, m)+r_{n}(l, m) P_{k-n}(l, m),
$$

where $q_{n}$ and $r_{n}$ are polynomials in $l^{ \pm 1}$ and $m^{ \pm 1}$. Then an easy induction on $n$ will prove

Lemma 8.3. If $k-n \geq 0$, then $B_{q_{n}}= \pm l^{n} m^{n}$ and $B_{r_{n}}= \pm l^{n+1} m^{n-1}$.

Proof. Since $q_{1}(l, m)=-l m$ and $r_{1}(l, m)=-l^{2}$, the lemma holds trivially for $n=1$. Now, from (8.4), we have

$$
\begin{aligned}
P_{k+1}(l, m)= & q_{n}(l, m)\left(-l m P_{k-n}(l, m)-l^{2} P_{k-n-1}(l, m)\right) \\
& +r_{n}(l, m) P_{k-n}(l, m) \\
= & \left\{-l m q_{n}(l, m)+r_{n}(l, m)\right\} P_{k-n}(l, m) \\
& -l^{2} q_{n}(l, m) P_{k-n-1}(l, m)
\end{aligned}
$$

and hence,

$$
q_{n+1}(l, m)=-l m q_{n}(l, m)+r_{n}(l, m) \quad \text { and } \quad r_{n+1}(l, m)=-l^{2} q_{n}(l, m) .
$$

Since, by the induction assumption, $B_{q_{n}}= \pm l^{n} m^{n}$ and $B_{r_{n}}= \pm l^{n+1} m^{n-1}$, we have $B_{q_{n+1}}= \pm m^{n+1} l^{n+1}$ and $B_{r_{n+1}}= \pm m^{n} l^{n+2}$. This completes the induction. 
Now (8.4) and Lemma 8.3 imply that

$$
P_{k+1}(l, m)=q_{k}(l, m) P_{1}(l, m)+r_{k}(l, m) P_{0}(l, m),
$$

where $B_{q_{k}}= \pm l^{k} m^{k}$ and $B_{r_{k}}= \pm m^{k-1} l^{k+1}$. We should note that $L_{0}$ is of the nonmultiple type or the connected sum of these, while $L_{1}$ is not of the nonmultiple type. Since $b_{l}(L), c(\widetilde{L})$ and $s(\widetilde{L})$ are additive w.r.t. a connected sum, by induction we have $b_{l}\left(P_{o}(l, m)\right)=c\left(\widetilde{L}_{0}\right)+s\left(\widetilde{L}_{0}\right)-1$. Since $c\left(\widetilde{L}_{0}\right)=$ $c(\widetilde{L})-k-1$ and $s\left(\widetilde{L}_{0}\right)=s(\widetilde{L})$, it follows that $b_{l}\left(P_{0}(l, m)\right)=c(\widetilde{L})+s(\widetilde{L})-$ $k-2$ and hence $b_{l}\left(r_{k}(l, m)\left(P_{0}(l, m)\right)=c(\widetilde{L})+s(\widetilde{L})-1\right.$. On the other hand, $L_{1}$ is not of the nonmultiple type, and hence (8.1) shows that $b_{l}\left(P_{1}(l, m)\right)<$ $c\left(\widetilde{L}_{1}\right)+s\left(\widetilde{L}_{1}\right)-1$. Since $c\left(\widetilde{L}_{1}\right)=c(\widetilde{L})-k$ and $s\left(\widetilde{L}_{1}\right)=s(\widetilde{L})$, it follows that $b_{l}\left(q_{k}(l, m) P_{1}(l, m)\right)<c(\widetilde{L})+s(\widetilde{L})-1-k+k=c(\widetilde{L})+s(\widetilde{L})-1$, and hence $b_{l}\left(P_{k+1}(l, m)\right)=b_{l}\left(r_{k}(l, m) P_{0}(l, m)\right)=c(\widetilde{L})+s(\widetilde{L})-1$, proving $(8.3)$.

Remark 8.1. It is not difficult to determine $b_{m}(L)$ for a prime special alternating link $L$ of the nonmultiple type. In fact, if there are exactly $q$ nontwigs in $\Gamma(\widetilde{L})$, then $b_{m}(L)=c(\widetilde{L})-3(s(\widetilde{L})-1)-2(q-1)$.

Example 8.1. Let $L$ be a pretzel link $L$ of type $\left(a_{1}, a_{2}, \ldots, a_{2 k}\right)$, where $a_{i} \geq$ 2 for all $i$. Then the orientation of $L$ can be chosen so that $L$ is a special alternating link of the nonmultiple type. Therefore $\mathbf{b}(L)=2 k$.

Appendix. Proof of Lemma 5.2. Let $L$ be an alternating fibered link and $\widetilde{L}=$ $L_{1} * \cdots * L_{h}$ a ${ }^{*}$-product representation of $L . L_{i}$ is an elementary torus link. $L_{i}$ spans an orientable surface $F_{i}$ consisting of two disks $D_{i, 1}$ and $D_{i, 2}$ which are joined by finitely many bands. Each band is half twisted positively or negatively. $L$ is constructed from these $L_{i}$ by a sequence of identifications of two disks $D_{i, 1}$ (or $D_{i, 2}$ ) and $D_{j, 1}$ or $D_{j, 2}$ [Mu1]. To illustrate this construction, we use a construction graph defined as follows. Take two vertices $v_{i, 1}$ and $v_{i, 2}$ on the plane associated with the disks $D_{i, 1}$ and $D_{i, 2}$ of $F_{i} \cdot v_{i, 1}$ and $v_{i, 2}$ are joined by an edge $e_{i}$. If $D_{i, 1}$, say, of $F_{i}$ is identified to $D_{j, 1}$, say, of $F_{j}$, then $v_{i, 1}$ and $v_{j, 1}$ associated with $D_{i, 1}$ and $D_{j, 1}$ are identified. The resulting graph $\Sigma(L)$ associated with $L$ is not only planar, but it is also a tree, since $L$ is fibered. (For more details, see [Mul].)

Now to construct a descending diagram $L_{0}$ from $L$, first define an order to the components $K_{1}, K_{2}, \ldots, K_{\mu}$ of $L$ and pick up a base point $b_{i}$ from each component $K_{i}$. Let $L_{0}$ be a descending diagram of $L$ w.r.t. this order and base points. $L_{0}$ represents a trivial link with trivial components $K_{1}^{\prime}, \ldots, K_{\mu}^{\prime}$. $L_{0}$ is obtained from $L$ by applying crossing changes at, say, $c_{1}, \ldots, c_{g}$. Let $\mathscr{S}=\left\{c_{1}, c_{2}, \ldots, c_{g}\right\}$. If $\mathscr{S}$ satisfies Lemma 5.2, we are done. If not, two cases can occur.

Case 1. None of the crossings on some $L_{j}$ belongs to $\mathscr{S}$.

Case 2. All crossings on some $L_{j}$ belong to $\mathscr{S}$. 


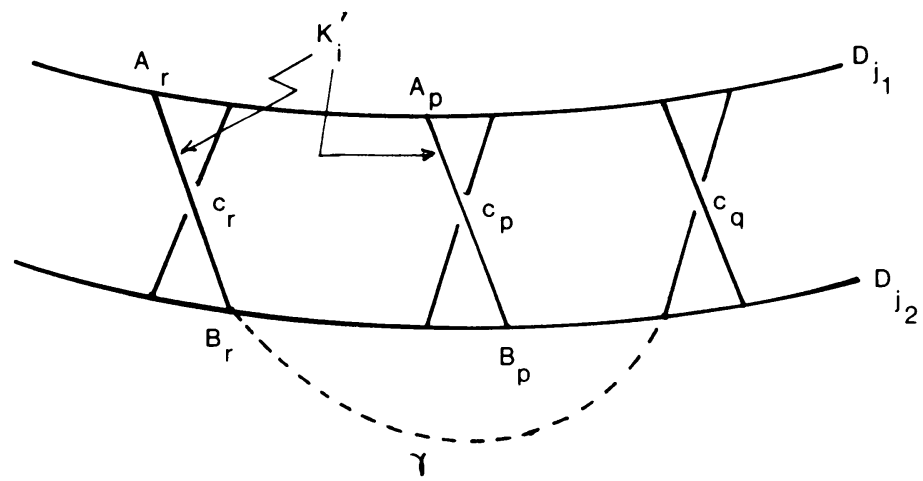

FIGURE 9

Since Case 2 is dual to Case 1, we consider only Case 1.

Let $K_{i}^{\prime}$ be the first component of $L_{0}$ that passes over crossings in $L_{j}$. Since no crossings on $L_{j}$ are in $\mathscr{S}$, no preceding component $K_{j}^{\prime}, j<i$, passes under crossings in $L_{j}$. Let $c_{r}$ be the first crossing on $L_{j}$ over which $K_{i}^{\prime}$ passes.

Suppose that $K_{i}^{\prime}$ crosses over another crossing $c_{p}$ on $L_{j}$, see Figure 9. Then $K_{i}^{\prime}$ must have a path $\gamma$ (in $L_{0}$ ) joining $B_{r}$ to $A_{p}$. Since the construction graph $\Sigma$ is a tree, $\gamma$ must cross under some crossing on $L_{j}$. Let $c_{q}$ be the first crossing on $L_{j}$ under which $\gamma$ passes. If $c_{q} \neq c_{r}$, then it follows from the choice of $K_{i}^{\prime}$ that a crossing change must be made at $c_{q}$ and hence $c_{q} \in \mathscr{S}$, a contradiction. Therefore, $c_{q}=c_{r}$, i.e., $c_{r}$ is a self-crossing of $K_{i}^{\prime}$. Then we make an unnecessary crossing change at $c_{r}$ without violating the property that $L_{0}$ be a trivial link. Now $\mathscr{S}^{\prime}=\mathscr{S} \cup\left\{c_{r}\right\}$ satisfies the condition in Lemma 5.2 for $L_{j}$.

Repeat this argument for $L_{t}$ when none of the crossings on $L_{t}$ belong to $\mathscr{S}$. This proves Lemma 5.2.

Added in proof. Conjecture A is false. A counterexample can be found in [M-P].

\section{REFERENCES}

[A] J. W. Alexander, A lemma on systems of knotted curves, Proc. Nat. Acad. Sci. U.S.A. 9 (1923), 93-95.

[B] J. Birman, Braids, links and mapping class groups, Ann. of Math. Studies, no. 82, Princeton Univ. Press, 1974.

[B-Z] G. Burde and H. Zieschang, Knots, De Gruyter, 1985.

[FYHLMO] P. Freyd, D. Yetter, J. Hoste, W. B. R. Lickorish, K. C. Millett, and A. Ocneanu, $A$ new invariant of knots and links, Bull. Amer. Math. Soc. 12 (1985), 239-246.

[G] D. Gabai, The Murasugi sum is a natural geometric operation, Contemp. Math. 20 (1983), 131-143.

[J] V. F. R. Jones, Hecke algebra representations of braid groups and link polynomials, Ann. of Math. 126 (1987), 335-388.

[K1] K. Kobayashi, On the genus of a link and the degree of the new polynomial. 
[K2] - On the minimum number of Seifert circles.

[L-M] W. B. R. Lickorish and K. C. Millett, A polynomial invariant of oriented links, Topology 26 (1987), 107-141.

[Mo] H. R. Morton, Closed braid representations for a link, and its Jones-Conway polynomial.

[Mu1] K. Murasugi, On a certain subgroup of the group of an alternating link, Amer. J. Math. 85 (1963), 544-550.

[Mu2] - On a certain numerical invariant of link types, Trans. Amer. Math. Soc. 117 (1965), $387-422$.

[Mu3] _ Jones polynomials of alternating links, Trans. Amer. Math. Soc. 295 (1986), 147-174.

[Mu4] _ Jones polynomials and classical conjectures in knot theory, Topology 26 (1987), 187194.

[Mu5] _ Jones polynomials and classical conjectures in knot theory (II), Math. Proc. Cambridge Philos. Soc. 102 (1987), 317-318.

[Mu6] _ On invariants of graphs with applications to knot theory, Trans. Amer. Math. Soc. 314 (1989), 1-49.

[M-P] K. Murasugi and J. H. Przytycki, An index of a graph with applications to knot theory.

[P] J. H. Przytycki, 3-braid representations of 2-bridge links.

[P-T] J. H. Przytycki and P. Traczyk, Invariants of links of Conway type, Kōbe J. Math. 4 (1987), 115-139.

[S] H. Schubert, Über eine numerische Knoteninvariante, Math. Z. 16 (1954), 245-288.

[Th] M. Thistlethwaite, A spanning tree expansion of the Jones polynomial, Topology 26 (1987), 297-309.

[Tr] P. Traczyk, Private communication.

[Y] S. Yamada, The minimal number of Seifert circles equals the braid index of a link, Invent. Math. 89 (1987), 347-356.

Department of Mathematics, University of Toronto, Toronto, Canada M5S 1A1 